\title{
TRANSPORTE-ME PARA O SEU LUGAR: O CASO DE SUCESSÃO FAMILIAR EM UMA EMPRESA DE TRANSPORTES
}

Carla Cazella ${ }^{1}$

Sayonara De Fátima Teston ${ }^{1}$

Carolina Matos ${ }^{2}$

leda Oro ${ }^{1}$

\footnotetext{
${ }^{1}$ Universidade do Oeste de Santa Catarina (UNOESC)

${ }^{2}$ Universidade do Vale do Itajaí
} 


\section{TRANSPORTE-ME PARA O SEU LUGAR: O CASO DE SUCESSÃO FAMILIAR EM UMA EMPRESA DE TRANSPORTES}

Resumo: O objetivo deste caso de ensino é despertar a reflexão sobre o tema sucessão familiar e cultura empreendedora.Chico, o protagonista deste caso, tem três filhos, e seu sócio tem dois filhos. Com o ingresso da segunda geração nos negócios, uma nova reestruturação foi necessária. Chico tornou-se o sócio majoritário. O caso oportuniza que o acadêmico assuma o papel do protagonista Chico para tomar decisões acerca da problemática apresentada, bem como, desabroche para percepção sobre a sucessão familiar, cultura empreendedora, os desafios atuais e a continuidade do empreendimento pelos filhos. O caso de ensino pode ser utilizado como material didático em cursos de graduação e de pós-graduação em administração e áreas afins. A elaboração do texto seguiu uma metodologia apropriada para caso de ensino. Os aspectos apresentados têm por objetivo possibilitar a discussão de conceitos sobre :Sucessão Familiar,Cultura Empreendedora e Empresas Familiares.

Palavras-chave: : Caso de Ensino; Sucessão Familiar; Cultura Empreendedora;Empresas Familiares.

Palavras-chave: até quatro, separadas por ponto (.).

\section{$1 \quad$ Introdução}

A empresa caso deste estudo é a Transportes Cordenonsi, localizada na cidade de Xaxim, Santa Catarina, foi fundada em 1982. É uma das pioneiras no transporte internacional e está presente em cinco países da América do Sul, entregando segurança, tecnologia e compromisso com a integridade da sua carga. Permanecer entre os grandes players do mercado é fruto de um trabalho competente, construído ao longo de décadas, que alçou a Cordenonsi à condição de parceira estratégica dos seus clientes para aproximar fronteiras e expandir negócios.

A Cordenonsi atende às principais empresas varejistas, alimentícias e de bens de consumo com transporte de cargas secas ou com temperatura controlada nas regiões Sul, Sudeste e parte da região Nordeste e no Mercosul. As operações contam com veículos de última geração, informações em tempo real para o cliente e acessibilidade total à equipe operacional. Vem de destacando entre as empresas pioneiras neste segmento, atendendo o 
Mercosul desde 1995, especificamente Argentina, Brasil, Chile, Paraguai e Uruguai. Atualmente é reconhecida pela experiência na gestão de cargas entre fronteiras e por nossa dedicação ao cliente.

A empresa está sediada na cidade de Xaxim e possui filiais em São Paulo - São Paulo, São José dos Pinhais - Paraná, Uruguaiana - Rio Grande do Sul, Estiva - Minas Gerais, Buenos Aires (Argentina), Santiago (Chile) e Montevidéu (Uruguai). Conta com 311 colaboradores, e é uma empresa familiar.

\section{Contexto do Caso: dados históricos}

Os pais de Luiz Alberto chegaram em Xaxim, Santa Catarina, em 1957 para trabalhar e residir. A família adquiriu um caminhão para transportar insumos de frigoríficos para São Paulo e trabalhou nesta atividade até 1966. Desde a infância, Luiz Alberto [fundador], também conhecido como Chico, relata que o pai chegava de viagem com "cheiro de poeira, de suor, eu adorava aquilo lá sabe, tão gostoso, que saudade", demonstrando que sua afeição pela área de transportes iniciou desde a infância.

Apesar do gosto pelo setor de transportes, em 1966, a família do Luiz Alberto juntamente com um sócio não familiar realizou a aquisição de uma distribuidora de bebidas. Apesar das dificuldades financeiras em que a empresa de distribuição de bebidas se encontrava, o pai do Chico, empreendedor, optou por parar de viajar para investir no novo negócio. Chico começou a trabalhar na distribuidora na área de produção, executando atividades de engarrafamento de bebidas, nesta época, com 9 anos de idade. Após 3 anos, passou a trabalhar como distribuidor nesta empresa e com sua bicicleta percorria a cidade e visitava os clientes. Posteriormente e com o crescimento das vendas, Chico fazia a distribuição no centro da cidade dirigindo uma pickup. Neste período, seu irmão mais velho, fazia a distribuição de bebidas no interior do município.

Incentivado principalmente pelo pai, Chico deu continuidade aos estudos. Em 1975, cursou o técnico em Contabilidade, e em 1976, ingressou no ensino superior noturno, no curso de Administração. Durante o curso de graduação, Chico precisava se deslocar para a cidade vizinha para estudar. No primeiro ano, era passageiro de um ônibus escolar, do qual se 
tornou motorista no ano seguinte. Esta atividade foi conciliada com as atividades de vendedor na empresa da família no período diurno. Em 1980, Chico concluiu o curso de graduação e nesta época as empresas da família estavam estruturadas e em franco desenvolvimento.

Chico possui outros irmãos, e com um deles trabalha até os dias atuais. "Sempre fui mais arrojado, meu irmão mais conservador [...] meu irmão é muito organizado, detalhista, eu sou mais do comercial [...] e sempre teve confiança e bom relacionamento entre nós", a complementaridade propiciou o consenso em investir em uma distribuidora de bebidas na cidade de Concórdia, Santa Catarina. De acordo com Chico, com o passar do tempo, a indústria fornecedora criou dificultadores para distribuidoras de bebidas que trabalhavam com diversas marcas, limitando as possibilidades de crescimento, e causando uma divisão nos lucros. Diante deste contexto e também para alegria da matriarca da família que desejava todos os filhos próximos, residindo em Xaxim, esta empresa de bebidas foi vendida. Com a venda, a família se capitalizou significativamente, o que possibilitou novos investimentos.

Percebendo a expansão das agroindústrias regionais, em 1982 a família voltou a investir no setor de transportes, iniciando com a aquisição de três caminhões. Chico menciona que era possível perceber que "o transporte é o termômetro da economia", tornando o setor atrativo para investimentos naquela época.

A partir de 1984, todos os anos, a empresa da família adquiria um novo caminhão e fazia investimentos em postos de combustíveis e imóveis. Em 1992, a família optou por vender todas as distribuidoras de bebidas, e após o falecimento dos pais, Chico e seus irmãos promoveram mudanças na empresa, e os dois irmãos sócios focaram seus investimentos na transportadora, expandindo oportunidades para outros estados do Brasil.

A partir de 1996, o crescimento regional das agroindústrias também propiciou o ingresso da empresa no Mercosul. Desde a infância, os filhos dos dois sócios foram gradativamente convivendo, aproximando-se e inseridos na empresa. Os membros da segunda geração aproximaram-se de maneira informal da empresa, quando ainda brincavam no pátio e conversavam de forma descontraída com os motoristas. Chico tem três filhos, e seu sócio tem dois filhos. Com o ingresso da segunda geração nos negócios, uma nova reestruturação foi necessária. Chico tornou-se o sócio majoritário. 
Com aproximadamente 15 anos de idade os dois filhos mais velhos de Chico, Fernando e Felipe, ingressaram na empresa e trabalharam em diferentes setores até assumir uma gerência. O filho mais novo de Chico, Luiz, foi inserido aos 18 anos, após voltar de um curso técnico, e atualmente, igual aos irmãos ocupa uma função de gestão. Chico procurou atribuir responsabilidades e dar "a oportunidade para errar, para que eles fossem aprendendo, tivessem autonomia". Chico também menciona a importância da troca de opinião intrageracional, os filhos trocam opiniões com o pai, tem espaço para se expressar e também assumem o erro junto quando algo não sai como esperavam. Chico também demonstra preocupação com a estrutura para uma vida pessoal confortável para os filhos: "dei um carrinho para cada um [...] agora todos têm salário [...] ajudei a fazer a casa depois que casaram", menciona Chico.

\section{Filhos ou funcionários? Pra quem vou entregar meu negócio?}

O filho mais velho de Chico, Fernando, é responsável pela gestão de negócios; o filho do meio, Felipe, atua da gestão de frotas e na área de Gestão de Pessoas; o filho mais novo, Luiz Filho, trabalha com atividades relacionadas à área administrativa, enquanto Chico ficou com a gestão estratégica. Apesar do arrojo, Chico preza por alguns valores, como "sempre administrar pela razão e não pela emoção", menciona que apesar de ter uma história que preza pela inovação, procura ter os pés "firmes no chão", afirmando que a tomada de decisões sobre investimentos precisa prezar pela segurança "o que, por que e pra quê vamos comprar algo?".

A estrutura societária da empresa dos três irmãos que integram a terceira geração é de $10 \%$ para cada um, enquanto Chico possui $35 \%$, assim como o sócio da mesma geração. Os dois filhos mais velhos possuem o mesmo salário, enquanto o filho mais jovem possui um salário um pouco menor. O filho mais jovem entrou por último na empresa e ainda está em processo de construção de sua legitimação, o que justifica o salário menor em comparação aos irmãos. Chico quer que todos os filhos continuem na empresa e contribuam com a sustentabilidade da organização.

Fernando, o filho mais velho, aproximou-se da empresa desde a infância, mas ingressou no processo de trabalho principalmente após os 15 anos. Executou atividades operacionais no início, mas desde seu ingresso, trabalhou ao lado do fundador, o que 
demonstra expectativa de ambos para assumir a gestão da empresa. $\mathrm{O}$ trabalho ocupa um papel prioritário na vida de Fernando o que faz com que a expectativa em relação aos demais funcionários e sócios seja alta no que diz respeito ao comprometimento e envolvimento com as atividades laborais.

Fernando demonstra assumir as responsabilidades de forma comprometida. Entre as qualidades, verifica-se ambição, bem como características visionárias, o que contribuiu para o crescimento da transportadora. Estes atributos trazem grande gratificação pessoal e profissional. Buscar inovação e novas tecnologias é um comportamento constante, o que também tem contribuído para a profissionalização do negócio. Gosta de atividades dinâmicas, demonstra iniciativa, aprecia desafios e tende a um comportamento mais propenso ao risco, e suas habilidades o permitem contribuir com o fomento da tomada de decisões, pois estão evoluindo na profissionalização dos processos de trabalho e consequentemente, na forma de gestão da empresa. No entanto sugere-se prudência no tratamento com os demais membros da segunda geração, uma vez que apesar de apresentarem diversas características comuns, também possuem diferenças individuais significativas. Olhares voltados para setores específicos e diferenças entre os sócios podem ser benéficas porque há complementação. Por outro lado, podem ocasionar conflitos intersetoriais.

Felipe, o filho do meio, aproximou-se da empresa desde a infância e das atividades com os caminhões na adolescência. A função de Felipe na gestão está voltada a atividades principalmente relacionadas às áreas de manutenção e de gestão de pessoas. Gerencia uma equipe interna no setor administrativo de 48 pessoas, e a equipe dos motoristas que são 246 pessoas. Portanto, o total de funcionários da empresa, que são 311 pessoas, encontra-se direta ou indiretamente ligados à gestão de Felipe. Felipe demonstra habilidade para lidar com pessoas, flexibilidade, habilidade de comunicação, comprometimento. Tende a uma postura centralizadora na execução das tarefas e demonstra um bom relacionamento com os demais sócios, assumindo uma postura adaptativa, o que é indicativo de inteligência social e emocional. Por meio das suas habilidades, busca contribuir para a distribuição de responsabilidades de forma tão igualitária quanto possível entre os membros da segunda geração.

. 
Luiz, o filho mais novo, aproximou-se da empresa principalmente após os 18 anos, pois esteve afastado do município para frequentar colégio agrícola. Demonstra ser mais introspectivo no trato social e mais objetivo na comunicação. Mudou de função recentemente na empresa e está iniciando sua inserção na gestão, principalmente em relação ao conhecimento/acesso às informações estratégicas. Luiz apresenta um perfil dinâmico, busca diversificar seus investimentos também para outros negócios, mas considera a Transportes Cordenonsi como a empresa onde quer manter seu maior foco, já que é através dela que obtém maior retorno financeiro. Luiz demonstra inteligência, com múltiplas soluções para os problemas que lhe são apresentados.

Chico reconhece que todos os filhos possuem características e habilidades diferentes e que todos podem contribuir muito com a empresa. Demonstra assim, interesse para que a sucessão se consolide.

\section{Dilema do caso}

O caso de ensino apresenta uma situação em que o fundador e proprietário de uma empresa familiar de grande porte do setor de transportes, precisa decidir por sair da gestão da empresa: Pra quem vou entregar meu negócio? Mas ainda está em dúvida se está preparado para isso e se seus sucessores internalizaram a cultura empreendedora que ele tanta prima. Chico sempre apresentou a preocupação pelas práticas de gestão adotadas pela empresa bem como o grau de profissionalização dentro da organização.

\section{Notas de ensino}

O objetivo deste caso de ensino é despertar a reflexão sobre o tema sucessão familiar e cultura empreendedora, tema ainda pouco pesquisado na literatuta atual. $\mathrm{O}$ caso oportuniza que o acadêmico assuma o papel do protagonista Chico para tomar decisões acerca da problemática apresentada, bem como, desabroche para percepção sobre a sucessão familiar, cultura empreendedora, os desafios atuais e a continuidade do empreendimento pelos filhos. O caso de ensino pode ser utilizado como material didático em cursos de graduação e de pósgraduação em administração e áreas afins. A elaboração do texto seguiu uma metodologia 
apropriada para caso de ensino. Os aspectos apresentados têm por objetivo possibilitar a discussão de conceitos sobre Empresa Familiar, Sucessão e Cultura Empreendedora.

\section{Fonte dos dados:}

As informações descritas no texto foram coletadas a partir de entrevistas junto ao proprietário e sucessores da empresa, totalizando quatro entrevistas. Trata-se de um caso baseado em uma história real e devidamente autorizado pela empresa participante. As entrevistas foram gravadas e transcritas na íntegra.

As informações contidas neste caso de ensino foram obtidas por meio de fontes de dados primárias, sendo essas obtidas por entrevista em profundidade. Dados secundários também podem ser utilizados pelos alunos para implementar a discussão. Para tal, sugere-se que os alunos realizem uma busca no site oficial da empresa (http://www.cordenonsi.com.br/) e em suas redes sociais. O caso é baseado em fatos reais, no entanto, a narrativa foi adaptada para fosse apresentada de forma mais atrativa aos leitores.

\section{Cursos indicados para utilização do Caso:}

O caso foi elaborado para aplicação principalmente com alunos de graduação nas áreas de Administração ou MBA (Master Business Administration), ou áreas afins, preferencialmente nas disciplinas de empreendedorismo, gestão empresarial, administração estratégica, teoria das organizações, psicologia organizacional e do trabalho, comportamento organizacional, governança, entre outras. Espera-se que o aluno, por meio do caso, desenvolva um entendimento sobre competências empreendedoras inseridas em um contexto de sucessão familiar, sob a ótica da aplicação dos conceitos teóricos na prática administrativa.

\section{Objetivos de Aprendizagem:}

Podem-se destacar alguns temas de aprendizagem:

a) Verificar quais as possibilidades de mudanças e analisar possíveis soluções para o dilema. 
b) Proporcionar discussões sobre os conceitos de cultura empreendedora em uma empresa de gestão familiar.

c) Promover reflexões sobre situações que permitam aos alunos analisar dilemas do processo de gestão de empresas familiares.

d) Debater sobre a realidade enfrentada por empresas familiares frente às dificuldades administrativas no decorrer de um processo de sucessão.

e) Compreender as diferentes perspectivas e cultura empreendedora em empresas familiares.

\section{Questões para discussão}

1) Você entende que os filhos de Chico têm potencial empreendedor? Quais características podem ser apresentadas para exemplificar sua resposta?

2) Qual(is) característica(s) de cada um dos sucessores mais se assemelha(m) às atitudes empreendedoras do seu pai?Identificaram Cultura empreendedora ou Cultura familiar?

3) Quais competências empreendedoras podem ser destacadas nos sucessores e como elas podem auxiliar no decorrer do processo de sucessão?

4) De que forma você identifica a cultura empreendedora ou cultura familiar neste caso?

5) De que forma é possível identificar as inovações na área da gestão introduzidas ao longo dos anos? Você entende que tais inovações vieram através da inclusão dos sucessores na empresa?

\section{Sugestões para o plano de aula:}

A análise do caso de ensino, baseado nas características da história do protagonista, proporcionam elementos para reflexão e discussão (Comin, Sabadin, \& Severo, 2017), que poderão auxiliar nos temas de aprendizagem.

Como sugestão para a compreensão e análise do caso pelo professor, para orientar a discussão em sala de aula, aconselham-se os seguintes passos: 
a) Preparação individual com pesquisa sobre a empresa no site oficial da empresa e nas redes sociais, bem como, realização de leitura prévia do caso por parte dos alunos.

b) Recomenda-se que o professor disponibilize um período para que os alunos discutam o caso em pequenos grupos.

c) Apresentação de cada grupo com orientação da discussão pelo professor.

d) Fechamento da discussão do caso retomando principais aspectos, com vistas a associar o caso com a teoria.

\section{Abordagens teóricas recomendadas}

Para aplicação deste caso pelos docentes, os autores sugerem algumas teorias de suporte, que servirão durante o processo de discussão e aprendizagem. Neste item serão abordadas teorias relacionadas a empresas familiares e sucessão e cultura empreendedora.

\section{Empresas familiares e sucessão}

O tema empresas familiares demonstra significância e importância na contemporaneidade, em níveis nacionais e internacionais e pode ser comprovado pela concentração desse tipo de organização na sociedade e na economia. De acordo com relatório da KPMG do Brasil (2018), as empresas familiares em todo mundo representam $70 \%$ do PIB Global e possuem uma série de características que as diferencia das demais empresas.

Dados do Sebrae indicam que entre $65 \%$ e $80 \%$ das empresas no mundo e pelomenos $80 \%$ das empresas brasileiras legalmente constituídas podem ser classificadas como familiares, ou seja, têm membrosde uma mesma família em seu controle acionário e, na maioria das vezes, também no comando da gestão. Inclusive,_quando se considera o total das empresas privadas brasileiras,as organizações familiares são responsáveis por mais de $61 \%$ da receita delas e por pelo menos dois terços dos empregos oferecidos.

Segundo Bellet et al. (2002), as pesquisas sobre as empresas familiares iniciaram na década de 80 e vêm crescendo ao longo dos anos. Pesquisadores buscam entender vários fatores que permeiam as empresas familiares, dentre inúmeros outros, quatro aspectos vem se 
destacando: sistemas de gestão; sucesso ou insucesso; processo sucessório e suas conseqüências e a relação com o tema do empreendedorismo (grifo nosso).

Pesquisadores descrevem que em países desenvolvidos, tendo como exemplo os Estados Unidos, onde pesquisas em empresas familiares são constantes, que as mesmas são significativas para o desenvolvimento social e econômico do país. Alguns dados são latentes, como os descritos no Quadro 1:

Quadro 1 - Características das empresas familiares

\begin{tabular}{|l|l|}
\hline \multicolumn{1}{|c|}{ Característica } & \multicolumn{1}{c|}{ Autor } \\
\hline $\begin{array}{l}\text { 90\% das empresas são familiares, participam com cerca de } \\
\text { 50\%, contribuem com 50\% na geração de empregos e são } \\
\text { responsáveis pela oferta de 9 em cada 10 novas ofertas de } \\
\text { emprego }\end{array}$ & $\begin{array}{l}\text { Bellet } \text { et al., (2002); Mccan III e } \\
\text { Leon-Guerreiro (2001); Reece (2003) } \\
\text { White, Krinke e Geller (2004). }\end{array}$ \\
\hline Metade das empresas familiares tem 60 anos ou mais & Fleming (1997). \\
\hline $\begin{array}{l}\text { Aproximadamente 30\% sobrevivem na segunda geração e } \\
\text { aproximadamente 10\% sobrevivem na terceira geração }\end{array}$ & $\begin{array}{l}\text { Fleming (1997), Avila e Naffziger } \\
\text { (2003) White, Krinke e Geller (2004). }\end{array}$ \\
\hline
\end{tabular}

Fonte: as autoras (2019)

A questão da sucessão é um tema recorrente na pauta das empresas familiares, fato que motiva a iniciativa de aprimorar o entendimento a respeito do comportamento desse tipo de fenômeno e dos elementos que o constituem, bem como das contingências que emergem da sua ocorrência nas organizações (Teston \& Fillippim, 2016).

A pesquisa sobre a questão sucessória em empresas familiares é fundamental para o entendimento sobre a natureza dessas organizações. A empresa familiar difere de outros tipos de organização principalmente pelo fato de haver em si, de forma intrínseca, a expectativa de perpetuidade, nutrida não somente no ambiente empresarial, mas também no seio familiar.

De acordo com Teston, Fillippim e Bencke (2016), diante da expressividade da questão da sucessão na empresa familiar, em termos de contribuição para o desenvolvimento do país, percebe-se a necessidade de aprimorar o entendimento a respeito desse tipo de organização e dos elementos que a constituem, bem como das contingências que emergem de sua dinâmica interna. Aprender para estar preparado pode ser visto como um indicador para os sucessores que estão na caminhada para assumir a gestão. É nessa linha de discussão que este artigo se insere, buscando encontrar alternativas e respostas aos questionamentos que emergem do debate. 
Existem diversos conceitos sobre a definição de empresa familiar, um dos mais utilizados no Brasil é o defendido por Bernhoeft (1991) que considera as empresas administradas há pelo menos duas gerações por uma mesma família e que mantenham membros dela na administração. Já Garcia (1997) considera, no seu conceito de empresa familiar, as organizações que podem ou não ter representantes na gestão, mas que, em contrapartida, exercem influências na missão e objetivos da organização a partir de interesses próprios.

O posicionamento do negócio familiar no contexto brasileiro realça sua importância estratégica, não apenas no âmbito socioeconômico interno mas também sob o ponto de vista do comércio exterior: $12 \%$ das empresas se dedicam à área de serviços, $34 \%$ à atividade industrial e 54\% se concentram no agribusiness (Ricca Neto, 2002), sendo esta última, reconhecidamente, uma das atividades na qual o País é internacionalmente competitivo e, por isso, uma das principais geradoras de divisas. A vantagem adicional desse fato é que tais divisas permanecem na economia brasileira, uma vez que, considerando tratar-se de empresas nacionais, numa situação normal não há remessa de seus lucros para o exterior.

Leone (2004) afirma ser internacionalmente aceito que as empresas familiares possuem três principais características: propriedade: o controle sobre as decisões está nas mãos da família;gestão: os mais elevados níveis hierárquicos são ocupados por membros da família e sucessão: a segunda geração da família assume os lugares vagos de seus antecessores familiares e, assim, sucessivamente.

Conforme afirma Sharma (2001), o processo de sucessão é definido como as ações, eventos e acontecimentos que afetam a transferência do poder de um membro da família para outro na organização. Esse processo inicia quando o dono da empresa demonstra a intenção que a sucessão ocorra até o momento em que o sucedido deixa o cargo para que outro indivíduo assuma sua posição (Massis, Chua, \& Chrisman, 2008).

Dyck et al. (2002) consideram que a sucessão pode representar uma oportunidade estratégica para organizações, especialmente quando se considera a dinamicidade do mercado. Nesse sentido, Machado (2003) enfatiza que a transição pode ser favorável, na medida em que provavelmente será acompanhada de algumas mudanças, em contraposição à tendência das empresas familiares em não processar mudanças no seu modo de gestão. Goffee (1996) 
considera que o êxito do processo sucessório está também ligado à habilidade do fundador em criar e comunicar uma cultura empresarial, embora os empreendedores, em geral, não estejam acostumados a delegar.

\section{Cultura Empreendedora}

Nesta seção trataremos do tema Cultura Empreendedora, mas para a base de entendimento buscou-se na antropologia primeiramente um conceito de Cultura que remete ao tema do estudo em tela. Optou-se como delineador do que se pretende evidenciar, o conceito de cultura do antropólogo Kluckhohn (1951), pois se pretende debater sobre as características das empresas familiares e cultura empreendedora originadas dos valores e crenças de seu fundador, de um líder que deixa sua marca.

Segundo Kluckhohn (1951), cultura consiste nas formas padronizadas de pensamento, sentimento e reação, adquiridas e transmitidas principalmente por símbolos, constituindo as realizações distintas de grupos humanos [...]; a essência da cultura consiste de crenças tradicionais (i.e. historicamente derivadas e selecionadas) e especialmente de seus valores incorporados.

De acordo com Colle, Oro e Carvalho (2017), as empresas possuem características, culturas diferentes e também desempenham funções distintas, de acordo com as suas necessidades. Para entender as empresas familiares, é necessário desenvolver mais conhecimento sobre culturas organizacionais subjacentes (Howorth, Rose, \& Hamilton, 2006). Cultura organizacional refere-seàs crenças compartilhadas, normas e valores mantidos coletivamente por pessoasem uma organização (por exemplo, uma família ou uma empresa).

Como citam Discua Cruz, Hamilton e Jack (2012), pesquisas recentes demonstram crescente interesse na compreensão da cultura, ou a maneira como as coisas são feitas, por trás dsa atividades empreendedoras em empresas familiares (Zahra, Hayton, \& Salvato, 2004). Os autores destacam que trabalhos anteriores destacam que muitas vezes podemos encontrar cultura organizacional paternalista e centrada no fundador quando atividades empreendedoras são engajadas. Tal visão enfatiza o próprio empreendimento familiar.

Zahra et al. ( 2004) destacam que pesquisas anteriores registram que as culturas empreendedoras sofrem influência da disposição das empresas existentes para apoiar e sustentar empreendedorismo . Embora os conceitos sejam diferentes, prevalece o consenso de 
que o empreendedorismo lida com os indivíduos e equipes envolvidas na identificação, avaliação e exploração oportunidades (Shane \& Venkataraman, 2000). Um empreendedor e a cultura devem então fomentar e apoiar a identificação contínua e busca de oportunidades que levem a continuidades de empreendimentos, sendo criados ou adquiridos. Empresas familiares foram encontradas para encorajar atividades empresariais ao longo do tempo (Kellermanns \& Eddleston, 2006; Webb, Ketchen, \& Ireland, 2010).

No entanto, a identificação e a busca de oportunidades geralmente estão associadas aos negócios fundadores da empresa familiar, tem sido questionado se as gerações sucessivas podem conseguem movimentar a cultura empreendedora que existia na geração fundadora (Westhead, Howorth, \& Cowling, 2001). Enquanto estudos destacam a importância dos fundadores no acúmulo de culturas empreendedoras (Chirico \& Nordqvist, 2010; Hall, Melin, \& Nordqvist, 2001) há uma compreensão limitada de como culturas empreendedoras são transmitidas e sustentadas.

Para Discua Cruz, Hamilton e Jack (2012), a influência da família fornece a base para a cultura do negócio. A cultura influenciada pela família define o que a família quer alcançar e como quer alcançá-la. Assim fazendo, a missão da família estabelece vieses, limites e possibilidade sobre o que a família almeja para o negócio.

\section{Considerações e instigação ao debate em sala}

O processo sucessório pode influenciar diretamente a continuidade da empresa, também afetada por outras variáveis importantes. Por essa razão, não deve ser tratado isoladamente, e sim em nível macro, a partir de uma reavaliação do contexto.

De acordo com Dupas et al. (2003), existem amplas evidências de que renunciar ao poder gerencial pode ser dolorosamente difícil, particularmente para o dirigente fundador. Para Ricca Neto (2002), os pontos críticos de uma sucessão estão no treinamento; avaliação na escolha dos sucessores; comparação do perfil do sucedido com o do sucessor, em relação ao que é exigido pela empresa; associação do sucesso da empresa com a imagem do fundador.

Percebe-se que a sucessão é uma fase das empresas familiares em que diversos conflitos surgem ou se tornam mais evidentes e, muitas vezes, os sucessores não sabem qual é a melhor maneira de conduzi-la. Ressalta-se a importância da organização em possuir, nesse 
momento, um planejamento adequado da sucessão para minimizar os problemas que podem advir, decorrente dessa passagem de poder, facilitando, consequentemente, esse processo.

Neste caso de ensino, quanto ao conceito de empresa familiar: considera-se empresa familiar aquela cuja gestão seja conduzida por um ou mais membros da família detentora do poder - controle societário, considerando que a existência de determinadas características no modelo de gestão permite à empresa familiar melhores condições de obter êxito em sua gestão e garantir a continuidade do negócio vamos ajudar o protagonista Chico neste dilema.

\section{Referências}

Avila, Stephen M.; Avila, Ramon A.; Naffziger, Douglas W. A Comparison Of FamilyOwned Businesses: Succession Planners And Nonplanners. Journal Of Financial Service Professionals.

Bellet, W.; et al. Family Business as a Field of Study. The Task Force of International Family Business Program Association, 2002

Bernhoeft, Renato. Empresa Familiar: sucessão profissionalizada ou sobrevivência comprometida. 2. ed.São Paulo: Nobel, 1991.179p

Chirico, F., \& Nordqvist, M. (2010). Dynamic capabilities and trans-generational value creation in family firms: The role of organizational culture. International Small Business Journal, 28(5), 487-504.

Colle, Diara; Oro, Ieda ; Carvalho, Carlos Eduardo. Influência da família no processo de internacionalização e desempenho nas empresas familiares. XX SEMEAD novembro de 2017 ISSN 2177-3866

Comin, Lidiane Cassia; Sabadin, RaquelKraemer; Severo, Eliana Andréa .Da vocação ao empreendedorismo: o dilema da mudança de carreira. RAT 2017

Dyck ,B., Mauws, M., Starke, F.A. \& Misschke,A. Passing the baton. The importance of sequence, timing, tchnique and communication in executive succession. Journal of Business Venturing, 17, p.143-162, 2002 
Dupas, Gilberto; Lank, Alden G.; Aires, Antônio; Vries, Manfred K. De; Costa, Roberto T.

Da. Governando a Empresa Familiar. ÁLVARES, Elismar (coord.). Rio de Janeiro: Qualitymark, 2003

Discua Cruz, A., Hamilton, E., \& Jack, S. L. (2012). Understanding entrepreneurial cultures in family businesses: A study of family entrepreneurial teams in Honduras. Journal of Family Business Strategy, 3(3), 147-161.

Garcia, V. P. Desenvolvimento das famílias empresárias. Rio de Janeiro: Qualitymark, 1997.

Goffee, R. Understanding family businesses: issues for further research. International Journal of Entrepreneurial Behavior \& Research. Vol 2, 1, p. 36-48, 1996

Hall, A., Melin, L., \& Nordqvist, M. (2001). Entrepreneurship as radical change in the family business: Exploring the role of cultural patterns. Family Business Review, 14(3), 193-208.

Howorth, C., \& Ali, Z. A. (2001). Family business succession in Portugal: An examination of case studies in the furniture industry. Family Business Review, 14(3), 231-244.

Kluckhohn, Clyde. The Study Of Culture. In: The Policy Sciences. Lerner, Daniel; Lasswall, Harold D. (Editores). Stanford, Ca: Stanford University Press, 1951

Leone, Nilda M. C. P. Guerra. A empresa Familiar Brasileira e a Opinião de seus Dirigentes sobre o Processo Sucessório. In: Organização/Estratégia, ENANPAD 2004

Mccann III, Joseph E.; Leon-Guerrero, Anna Y. Strategic Goals And Practices Of Innovative Family Business. Journal Of Small Business Management

Machado, HilkaPelizza Vier. Sucessão familiar e gênero: implicações para estudos sobre empresas familiares. Encontro de estudos sobre empreendedorismo e gestão de pequenas empresas. 3., 2003, Brasília. Anais... Brasília: UEM/UEL/UnB, 2003.

Massis, A.; Chua, J.; Chrisma, J. J. Factorspreventingintra-familysuccession. Family Business Review, Massachusetts, v. 21, n. 2, Jun., 2008.

Reece, Ronald C. How To Mix Blood And Business Safely And Successfully. Journal Of Financial Service Professionals

Ricca Neto, Domingos. Quem são as empresas familiares? Abrac - Associação Brasileira de Concessionárias Chevrolet, São Paulo, 20 mar. 2003 
Sharma, P., Chrisman, J. J., Pablo, A., \&Chua, J. (2001). Determinant sofinitialsatisfactionwiththesuccessionprocess in family firms: a conceptual model. Entrepreneurship Theoryand Practice, 25(3), 17-36.

Shane, S., \& Venkataraman, S. (2000). The promise of entrepreneurship as a field of research. Academy of Management Review, 25(1), 217-226.

Smyrnios, \& S. Klein (Eds.), Handbook of research on family business (pp. 614-618). Cheltenham, UK: Edward Elgar

Teston, Sayonara de Fatima; Fillippim, Eliane Salete; Benck, Fernando Fantoni. Aprendendo A Ser Sucessor: Um Olhar Sobre A Experiência . Perspectivas em Gestão \& Conhecimento, João Pessoa, v. 6, n. 1, p. 155-174, jan./jun. 2016. http://periodicos.ufpb.br/ojs2/index.php/pgc. ISSN: 2236-417X.

Teston, Sayonara de Fatima; Fillippim, Eliane Salete. Perspectivas e Desafios da Preparação de Sucessores para Empresas Familiares RAC, Rio de Janeiro, v. 20, n. 5, art. 1, pp. 524545, Set./Out. 2016 http://dx.doi.org/10.1590/1982-7849rac2016150033

Webb, J. W., Ketchen, D. J., Jr., \& Ireland, R. D. (2010). Strategic entrepreneurship within family-controlled firms: Opportunities and challenges. Journal of Family Business Strategy, 1(2), 67-77.

Westhead, P., \& Wright, M. (1998). Novice, portfolio and serial founders: Are they different? Journal of Business Venturing, 13, 173-204

White, William S.; Krinke, Timothy D.; Geller, David L. Family Business Succession Planning: Devising An Overall Strategy. Journal Of Financial Service Professionals

Zahra, S. A., Hayton, J. C., \& Salvato, C. (2004). Entrepreneurship in family vs. non-family firms: A resource-based analysis of the effect of organizational culture. Entrepreneurship Theory and Practice, 28(4), 363-381.

Zahra, S. A., Klein, S., \& Astrachan, J. (2006). Epilogue: theory building and the survival of family firms - three promising research directions. In P. Poutziouris, K. 